\title{
Aspectos epidemiológicos asociados a toxocarosis en Gualeguaychú, Entre Ríos. Argentina
}

\author{
MARTA C. MINVIELLE*, MARÍA R. TAUS**, MARÍA L.CIARMELA*, MARIELA FRANCISCONI**, \\ MARIANA BARLASINA**, BETINA C. PEZZANI*, ALEJANDRA GASPAROVIC**, \\ ARGELIA RAFFO** y CARLOS GOLDARACENA**.
}

\section{EPIDEMIOLOGICALASPECTS ASSOCIATED TO TOXOCAROSIS IN GUALEGUAYCHÚ, ENTRE RÍOS, ARGENTINA}

The aim of the present estudy was to determine the epidemiological factors of toxocarosis in Gualeguaychú, Argentina. It was evaluated the seroprevalence of this parasitoses in 272 healthy blood donors (ELISA test), and the relationship of this parameter with the socioeconomic conditions of persons, presence or absence of parasitized dogs in their dwellings and sorroundings; and the contamination of publics places of this city with Toxocara spp ova. The seroprevalence was 12,13\% with most of the individuals being asymptomatic. Neither socioeconomic status nor dog ownership correlated significantly with positive serology. Toxocara canis eggs were found $25 \%$ of the dogs studied and in $100 \%$ of relevated public places. It can be therefore concluded that there is a high frequency of contamination in the soils of public places with $\boldsymbol{T}$. canis eggs, as well as a high percentage of dog infected with this nematode. This situation establishes an important risk to acquire toxocarosis for the inhabitans of this city.

Keys words: Toxocarosis, Zoonosis, Public Health, Epidemiology.

\section{INTRODUCCIÓN}

La toxocarosis humana fue descripta por primera vez bajo el nombre de larva migrans visceral ${ }^{1}$. Su agente etiológico más frecuente es Toxocara canis, un nematodo de caninos que accidentalmente infecta al hombre ${ }^{2}$.

Personas que nunca han tenido perros en su domicilio se han visto afectadas, lo que ha llevado a tomar conciencia sobre la contaminación ambiental con materia fecal canina parasitada, especialmente de paseos públicos, lugares donde juegan niños y calles de la ciudad ${ }^{3,4}$.

En Argentina, han sido reportados por diversos autores ${ }^{5,6}$ en los últimos años estudios sobre prevalencia de toxocarosis. En el presente trabajo se estudiaron los factores epidemiológicos asociados a esta parasitosis en Gualeguaychú, ciudad de 70.000 habitantes, en Entre Ríos, Argentina.

* Cátedra de Microbiología y Parasitología. Facultad de Ciencias Médicas. Universidad Nacional de La Plata, Argentina.

** Laboratorio de asesoramiento y control de Zoonosis Emergentes de esta región. Facultad de Bromatología. Universidad Nacional de Entre Ríos, Argentina.

Correspondencia a: Rosalba Taus. Cátedra de Química Biológica. Facultad de Bromatología, Universidad Nacional de Entre Ríos. 25 de Mayo 701 (2820) Gualeguaychú, Entre Ríos, Argentina 


\section{MATERIAL Y MÉTODOS}

Población humana y encuesta socioeconómica: se analizó la seroprevalencia de toxocarosis en 272 donantes de sangre voluntarios clínicamente sanos, de la ciudad de Gualeguaychú. Del total, $204(75 \%)$ fueron hombres y 68 (25\%) mujeres. La mayoría había residido toda su vida en este municipio y sus edades fueron de 18 a 75 años (र्X: 39 años).

Mediante una encuesta personal se recabaron datos sobre: localización de la vivienda, características de la misma: tipo de construcción, baño (completo, incompleto o no posee), modo de eliminación de las excretas, condiciones sanitarias, eliminación de la basura, suministro de agua, tenencia de mascotas en la vivienda y registro de signos y/o síntomas relacionados con la parasitosis.

Se analizaron 100 materias fecales de perros (Tabla 1) por el método de concentración por sedimentación de Telemann modificado y posterior observación microscópica de los huevos. También se analizaron las heces de los caninos pertenecientes a las personas que fueron seropositivos para toxocarosis y poseían perros en su domicilio $(n=15)$. Se recolectó una sola muestra de cada perro en formol al 5\%, las mismas fueron procesadas por el método de Telemann modificado y posterior observación microscópica entre porta y cubreobjetos.

Se analizaron muestras del suelo correspondientes a 7 paseos y 11 plazas de la ciudad. Se retiró la hojarasca superficial de un cuadrado de $20 \mathrm{~cm}$ de lado raspando no más allá de $\operatorname{los} 2 \mathrm{~cm}$ de profundidad. Cada muestra homogeneizada se trato con Tween 80 al 10\%, filtrando y luego decantando por 24 horas. El sedimento se procesó por dos métodos: 1) por la técnica de
Telemann modificada y 2) por un método de flotación con una solución saturada de nitrato de sodio, con posterior observación microscópica.

Los datos fueron evaluados estadísticamente por el método de $\chi^{2}$ considerando como diferencia significativa un valor de $\mathrm{p}<0,01$.

\section{RESULTADOS}

La seroprevalencia de toxocarosis fue de $12,13 \%$ (33/272), con $29(14,21 \%)$ de sueros positivos pertenecientes al sexo masculino y 4 $(5,88 \%)$ al femenino.

No se encontró correlación estadística entre las condiciones socioeconómicas y la seroprevalencia de esta patología. La mayoría de los individuos seropositivos manisfestaron no poseer síntomas asociados a toxocarosis.

Los hallazgos de huevos de T. canis en la materia fecal canina se presentan en Tabla 1. El análisis de materia fecal de los perros pertenecientes a los individuos seropositivos reveló una frecuencia del $20 \%$ (3/15).

La frecuencia total de contaminación de los paseos públicos con huevos de T. canis fue del $100 \%$ utilizando la técnica de sedimentación respecto al $28 \%$ usando el procedimiento de flotación.

\section{DISCUSIÓN}

La seroprevalencia de toxocarosis encontrada en este estudio en donantes de sangre es mayor a la reportada en poblaciones similares de diferentes países europeos ${ }^{7,8}$, pero menor que la descripta en indígenas de Venezuela9.

Un trabajo indica una mayor infección en las mujeres con respecto de los hombres ${ }^{10}$. En nuestro trabajo, los varones duplicaron su

Tabla 1. Presencia de huevos de Toxocara canis en materia fecal de 100 perros. Gualeguaychú. Entre Ríos

\begin{tabular}{lcccc}
\hline & Vag & C/dueño/calle & C/dueño/def pp & Totales \\
\hline Positivos & 11 & 8 & 6 & 25 \\
Negativos & 14 & 30 & 31 & 75 \\
Totales & 25 & 38 & 37 & 100 \\
$\%$ & 25 & 38 & 37 & 100 \\
\hline
\end{tabular}

Ref. vag.: vagabundo.

C/dueño/calle: con dueño que están en la calle sin control.

$\mathrm{C} / \mathrm{dueño/def} \mathrm{pp:} \mathrm{con} \mathrm{dueño} \mathrm{que} \mathrm{los} \mathrm{sacan} \mathrm{a} \mathrm{pasear} \mathrm{y} \mathrm{defecan} \mathrm{en} \mathrm{paseos} \mathrm{públicos.}$ 
porcentaje de seroprevalencia respecto de las mujeres. En este estudio no existió diferencia significativa entre los individuos positivos y negativos y las variables relevadas y tampoco respecto a la presencia / ausencia de caninos en el hogar. El 20\% (3/15) de los perros de las personas con anticuerpos antitoxocara estaban infectados, por lo que podríamos inferir que la posesión de mascotas en el hogar no constituyó un factor de riesgo en esta población. El relevamiento sobre 100 perros de la ciudad demostró una frecuencia del 25\% (25/100), porcentaje semejante al que se encontró en 500 perros con dueño en el Distrito Federal de Méjico ${ }^{11}$.

El suelo contaminado con huevos embrionados de $T$. canis es la principal fuente de infección humana $^{12}$. En nuestro estudio el $100 \%$ de los paseos públicos analizados por el método de Telemann modificado estaban parasitados por huevos de T. canis.

Del análisis de lo expuesto es indudable que la seroprevalencia de toxocarosis en esta población estaría asociada al elevado grado de infección por T. canis de los perros de este municipio (donde la recolección de los heces no es obligatoria por ley), que defecan en los paseos públicos y en las calles de la ciudad determinando la elevada contaminación del suelo encontrada.

Este estudio demuestra que la población de Gualeguaychú se encuentra expuesta a la infección por huevos de $T$. canis presentes en el suelo de paseos públicos. El registro de la seroprevalencia de toxocarosis en donantes de sangre confirma la infección con este parásito en esta ciudad.

\section{RESUMEN}

El objetivo del estudio fue determinar factores epidemiológicos de toxocarosis en la ciudad de Gualeguaychú, Entre Ríos, Argentina. Se evaluó en 272 donantes de sangre la seroprevalencia de esta parasitosis, su relación con las condiciones socioeconómicas de las personas estudiadas, presencia o ausencia de perros parasitados en sus hogares y en los alrededores; y la contaminación de plazas y paseos públicos con huevos de Toxocara spp. La seroprevalencia fue del 12,13\% sin correlacionarse significativamente con condiciones socioeconómicas ni tenencia de perros, siendo la mayoría de los individuos asintomáticos. El 25\% de los perros y el $100 \%$ de los paseos públicos estudiados, resultaron parasitados. Esta situación establece un riesgo importante de adquirir toxocarosis en los habitantes de esta ciudad.

\section{REFERENCIAS}

1.- BEAVER P C. Parasitological reviews: larva migrans. Exp Parasitol 1956; 5: 587-621.

2.- GLICKMAN L, SCHANTZ P, CYPESS R. Canine and human toxocariasis: review of transmission, pathogenesis and clinical disease. J Am Vet Med Assoc 1979; 175: 1265-69.

3.- HOLLAND C, O'CONNOR P, TAYLOR M et al. Families, parks, gardens and toxocariasis. Scand J Infect Dis 1991; 23: 225-31.

4.- UGA S, MINAMI T, NAGATA K. Defecation habits of cats and dogs and contamination by Toxocara eggs in public park sandpits. Am J Trop Med Hyg 1996; 54 : 122-6.

5.- ARCHELLI S, GUARDIS M, RADMAN N et al. Toxocarosis en niños en edad pre-escolar en la ciudad de La Plata. Libro de resúmenes del III Congreso Argentino de Parasitología, Mar del Plata, Tomo II, 2000, p: 385 .

6.- SZRETTER A, DALLA FONTANA M L, FUSCO $S$ et al. Prevalencia parasitológica en niños de la localidad de Rueda, departamento de Constitución, Santa Fe, Noviembre de 1999. Libro de resúmenes del III Congreso Argentino de Parasitología, Mar del Plata, Tomo II, 2000, p: 389.

7.- GENCHI C, DISACCO B, GATTI S et al. Epidemiology of human toxocariasis in northern Italy. Parasitologgia 1990; 32: 313-9.

8.- JIMÉNEZ J F, VALLADARES B, FERNÁNDEZ PALACIOS J M et al. A serologic study of human toxocariasis in the Canary Islands (Spain): environmental influences. Am J Trop Med Hyg 1997; 56, 113-5.

9.- LYNCH N, EDDY K, HODGEN A et. Al Seroprevalence of Toxocara canis infection in tropical Venezuela. Trans Royal Soc Trop Med Hyg 1988; 82: 275-81.

10.- MAGNAVAL J F, BAIXENCH M T. Toxocariasis in the Midi Pyrenées region. In: Toxocara and Toxocariasis, Lewis \& Maizels (editors). London: British Society for Parasitology. 1993; pp 63-69.

11.- MARTÍNEZ BARBABOSA I, FERNÁNDEZ PRESAS A M, VÁZQUEZ O, RUIZ HERNÁN-DEZ A. Frecuencia de Toxocara canis en perros y áreas verdes del sur de la ciudad de México, Distrito Federal. Vet Méx 1998; 29: 239-44.

12.- GLICKMAN L T. The epidemiology of human toxocariasis. In: Toxocara and Toxocariasis, Lewis \& Maizels (editors). London: British Society for Parasitology. 1993; pp 3-10. 